OPEN ACCESS

Edited by:

Fabio DiLisa,

University of Padua, Italy

Reviewed by:

Ove Eriksson,

University of Helsinki, Finland

Sonia Cortassa

National Institutes of Health, USA

*Correspondence:

Craig Porter

cr2porte@utmb.edu

Specialty section

This article was submitted to

Mitochondrial Research,

a section of the journal

Frontiers in Physiology

Received: 08 December 2016

Accepted: 23 February 2017

Published: 13 March 2017

Citation:

Rontoyanni VG, Nunez Lopez O,

Fankhauser GT, Cheema ZF

Rasmussen BB and Porter C (2017)

Mitochondrial Bioenergetics in the

Metabolic Myopathy Accompanying

Peripheral Artery Disease.

Front. Physiol. 8:141

doi: 10.3389/fphys.2017.00141

\section{Mitochondrial Bioenergetics in the Metabolic Myopathy Accompanying Peripheral Artery Disease}

\author{
Victoria G. Rontoyanni, ${ }^{1,2}$ Omar Nunez Lopez ${ }^{1,2}$, Grant T. Fankhauser', \\ Zulfiqar F. Cheema ${ }^{2}$, Blake B. Rasmussen ${ }^{3}$ and Craig Porter ${ }^{1,2 *}$ \\ ${ }^{1}$ Metabolism Unit, Shriners Hospitals for Children, Galveston, TX, USA, ${ }^{2}$ Department of Surgery, University of Texas Medical \\ Branch, Galveston, TX, USA, ${ }^{3}$ Department of Nutrition and Metabolism, University of Texas Medical Branch, Galveston, \\ TX, USA
}

Peripheral artery disease (PAD) is a serious but relatively underdiagnosed and undertreated clinical condition associated with a marked reduction in functional capacity and a heightened risk of morbidity and mortality. The pathophysiology of lower extremity PAD is complex, and extends beyond the atherosclerotic arterial occlusion and subsequent mismatch between oxygen demand and delivery to skeletal muscle mitochondria. In this review, we evaluate and summarize the available evidence implicating mitochondria in the metabolic myopathy that accompanies PAD. Following a short discussion of the available in vivo and in vitro methodologies to quantitate indices of muscle mitochondrial function, we review the current evidence implicating skeletal muscle mitochondrial dysfunction in the pathophysiology of PAD myopathy, while attempting to highlight questions that remain unanswered. Given the rising prevalence of PAD, the detriment in quality of life for patients, and the associated significant healthcare resource utilization, new alternate therapies that ameliorate lower limb symptoms and the functional impairment associated with PAD are needed. A clear understanding of the role of mitochondria in the pathophysiology of PAD may contribute to the development of novel therapeutic interventions.

Keywords: mitochondria, mitochondrial function, bioenergetics, peripheral artery disease, peripheral vascular disease, skeletal muscle

\section{INTRODUCTION}

Peripheral artery disease (PAD) is the third leading cause of cardiovascular morbidity after coronary heart disease and stroke (Fowkes et al., 2013). In the preceding decade, the global prevalence of PAD increased by $\sim 24 \%$, corresponding to an estimated 202 million people living with PAD worldwide (Fowkes et al., 2013). In the US alone, $\sim 8.5$ million adults aged $\geq 40$ years are affected by PAD (Allison et al., 2007; Mozaffarian et al., 2015), with annual hospitalization costs estimated at more than $\$ 21$ billion (Mahoney et al., 2008, 2010). Yet, diagnosis and treatment of PAD is often overlooked, and clinical trials focusing on PAD are relatively sparse considering the prevalence and impact of the disease (Jaff, 2014; Subherwal et al., 2014).

As an atherosclerotic occlusive disease, PAD results in obstruction of the conduit arteries serving the lower extremities. Reduced blood flow, and skeletal muscle and nerve abnormalities contribute to the functional impairment and limb manifestations associated with PAD. In primary 
care facilities, $30-60 \%$ of all PAD patients are diagnosed as asymptomatic, (i.e., no exertional leg symptoms), $\sim 10 \%$ exhibit classic symptoms of intermittent claudication (i.e., exertional calf pain that is induced by exercise/walking and subsides with rest), and the remainder present with atypical leg symptoms, i.e., exertional leg symptoms that are not consistent with classic intermittent claudication. Atypical leg symptoms commonly include leg pain on exertion and rest (i.e., exertional leg pain that initiates at rest but is not associated with critical limb ischemia), and leg pain/carry on (i.e., exertional leg pain that does not prompt the patient to stop exercising/walking; McDermott, 2015; Hiatt et al., 2015). Over a 5-year follow-up, 1-2\% of PAD cases will progress to critical limb ischemia (i.e., ischemic rest pain and tissue loss, such as skin ulceration and gangrene; Hirsch et al., 2006; McDermott, 2015). Irrespective of this classification, all patients with $\mathrm{PAD}$, even asymptomatic individuals, have reduced functional capacity (McDermott et al., 2000, 2008) and a heightened risk of morbidity and mortality (Diehm et al., 2009). Over time, functional capacity in PAD declines further, the degree of the change being associated with the severity of the disease (McDermott et al., 2004).

Individuals with PAD are less physically active (McDermott et al., 2002) and tend to have a maximal exercise capacity that is approximately half of that of age-matched healthy individuals (Hiatt et al., 1987). Further, patients with PAD who develop critical limb ischemia are at increased risk for limb loss. Strikingly, PAD accounts for $\sim 70,000$ major amputations performed annually within the U.S., with an estimated cost of $\$ 10.6$ billion (Yost, 2014). It is predicted that the number of allcause amputees living in the US will rise from 1.6 million to 3.6 million by 2,050, with PAD accounting for the majority of this projected increase (Ziegler-Graham et al., 2008). Moreover, PAD patients who require lower limb amputation have a 5-year mortality of over $50 \%$, a poorer prognosis to that of breast, prostate, and colon cancer patients (Armstrong et al., 2007; Robbins et al., 2008). Thus, PAD represents a significant problem in modern health care, in terms of its increasing prevalence, fiscal impact on health care systems, and detriment in quality of life for patients.

Considering the largely asymptomatic nature of PAD in its early stages (McDermott et al., 2000; Hirsch et al., 2006; McDermott, 2015), and the detrimental consequences of its progression, early clinical diagnosis, and effective management of PAD is imperative. The most cost-effective tool for early clinical diagnosis of lower extremity PAD is the ankle-brachial index $(\mathrm{ABI})$, which is the ratio of resting systolic blood pressure measured at the ankle (dorsalis pedis and posterior tibial arteries) to that at the brachial artery; an ABI value of $<0.90$ is $68-$ $84 \%$ sensitive and $84-99 \%$ specific for PAD (Aboyans et al., 2012; Gerhard-Herman et al., 2016). Major risk factors for PAD include advancing age, hypertension, smoking, diabetes mellitus, and hyperlipidemia (Fowkes et al., 2013). As with other forms of atherosclerotic vascular disease, the 2016 AHA/ACC guidelines for treatment of PAD recommend cardiovascular risk factor modification, in addition to PAD specific treatment for claudication and critical limb ischemia. Claudication is primarily addressed via exercise training, and administration of phosphodiesterase inhibitors for their antiplatelet and vasodilatory properties (Hirsch et al., 2006; Gerhard-Herman et al., 2016).

Effective long-term management of limb symptomatology and functional impairment in PAD depends on targeted therapies addressing the pathophysiology of the disease. While atherosclerotic occlusive disease, decreased blood perfusion, and restricted $\mathrm{O}_{2}$ delivery to skeletal muscle is a major contributor to the limb manifestations in $\mathrm{PAD}$, reduced blood flow only partially accounts for the functional deficit associated with PAD. Additional factors contribute to the pathophysiology of the functional impairment in PAD, with evidence suggesting abnormalities in skeletal muscle metabolism, where mitochondria may play a key role (Brass and Hiatt, 2000; Kemp, 2004; Pipinos et al., 2007). Accordingly, the aim of this review article is to explore and discuss the current evidence for a role of mitochondrial dysfunction in the metabolic myopathy observed in PAD patients. Given the rising prevalence of PAD and the societal impact that it incurs, a better understanding of the underlying determinants of reduced muscle function seen in PAD patients represents a critical step in the development of novel therapeutic interventions.

\section{PATHOPHYSIOLOGY OF LIMB MANIFESTATIONS AND FUNCTIONAL IMPAIRMENT IN PAD}

\section{Hemodynamic Abnormalities}

The sequelae of events leading to limited functional capacity in PAD patients originate from a hemodynamic deficit resulting from one or more arterial stenoses and/or occlusions in the iliac, femoral, popliteal, or tibial arteries (Hiatt et al., 2015). Blood flow distribution to tissues is relative to their metabolic activity. At rest, skeletal muscle perfusion represents $\sim 20 \%$ of cardiac output (McArdle et al., 2007). During exercise, blood flow is redistributed to the active skeletal muscles, with muscle blood flow accounting for up to $80-85 \%$ of cardiac output at high exercise intensities (McArdle et al., 2007). Although the presence of arterial stenosis or occlusion can adversely affect blood flow dynamics, in the absence of critical limb ischemia resting blood flow to lower extremities is preserved via compensatory mechanisms distal to the site of the occlusion, such as collateral blood supply (Bragadeesh et al., 2005; Traupe et al., 2013). However, in states of high metabolic demand, such as exercise, the arterial stenosis, and occlusions in PAD become flow limiting. Blood flow is further compromised by impaired peripheral vasodilation, as evidenced by macroand micro-vascular endothelial dysfunction (Joras and Poredos, 2008; Coutinho et al., 2011a; Grenon et al., 2014; Heinen et al., 2015), and increased arterial stiffness and pressure wave reflections in patients with PAD (Brewer et al., 2007; AmohTonto et al., 2009; Coutinho et al., 2011b; Beckmann et al., 2015). This flow limitation results in inadequate $\mathrm{O}_{2}$ delivery to the mitochondria of contracting skeletal muscle, limiting oxidative phosphorylation (Brass and Hiatt, 2000; Pipinos et al., 2000, 2003). 


\section{Skeletal Muscle Abnormalities}

Although reduced blood flow is a significant contributor to clinical limb manifestations as indicated by the association of low ABI with leg pain symptoms (Wang et al., 2005), leg blood flow and ABI appear to be no more than weakly associated with functional/exercise capacity (Pernow and Zetterquist, 1968; Szuba et al., 2006; McDermott et al., 2013; Nardi Gomes et al., 2015). Indeed, revascularization of occluded blood vessels does not fully restore the muscle functional limitations in PAD patients (Regensteiner et al., 1993a; Gardner and Killewich, 2001; West et al., 2012), while exercise treatment improves functional capacity with negligible (Larsen and Lassen, 1966; Sorlie and Myhre, 1978) to modest increases in leg blood flow (Hiatt et al., 1990). These findings indicate that additional factors besides hemodynamic limitations contribute to the pathophysiology of the functional impairment in PAD.

The mismatch between oxygen and substrate demand and delivery to active skeletal muscles during exercise via a blood flow reduction (exercise-induced ischemia) and the associated prolonged hyperemic effect in the post-exercise resting period (reperfusion) trigger a cascade of pathophysiological responses (Hiatt et al., 2015). During ischemia and reperfusion, there is a rise in cytosolic $\mathrm{Ca}^{2+}$ with subsequent mitochondrial $\mathrm{Ca}^{2+}$ overload owing to mitochondria's role as an intracellular $\mathrm{Ca}^{2+}$ buffer, and there is a concurrent burst of reactive oxygen species (ROS) generation (Murphy and Steenbergen, 2008). These events can trigger mitochondrial permeability transition pore opening, which can limit adenosine triphosphate (ATP) production and activate apoptotic signaling, ultimately leading to cell death (Murphy and Steenbergen, 2008). Indeed, there is evidence of elevated ROS production and associated oxidative damage in skeletal muscle of PAD patients, which may be exacerbated by a compromised antioxidant defense system (Bhat et al., 1999; Pipinos et al., 2006, 2008). Most notably, previous studies in PAD patients demonstrated increased mitochondrial DNA injury (Bhat et al., 1999) as well as elevated protein oxidation and lipid peroxidation (Pipinos et al., 2006). Mitochondrial DNA damage has been detected in both limbs of patients with unilateral PAD, suggesting a potential systemic effect (Bhat et al., 1999). Inflammation and oxidant stress are likely implicated in subsequent skeletal muscle structural and metabolic abnormalities associated with PAD, including mitochondrial dysfunction, muscle fiber degeneration, muscle fibrosis, and muscle apoptosis and atrophy (Regensteiner et al., 1993b; Brass and Hiatt, 2000; Mitchell et al., 2007; Pipinos et al., 2007; Koutakis et al., 2015). In PAD patients with intermittent claudication, $3.8 \%$ of gastrocnemius cells were determined as apoptotic vs. $1.5 \%$ in age-matched controls, and caspase- 3 activity (a key component of apoptosis which is activated by the mitochondrion) was double that in patients without PAD (Mitchell et al., 2007), suggesting that mitochondrial stress is linked to programmed cell death in skeletal muscle of PAD patients. The complex pathophysiology of exertional limb manifestations has been reviewed in detail by others (Pipinos et al., 2007, 2008; Hiatt et al., 2015).

\section{THE ROLE OF THE MITOCHONDRION IN PAD}

Mitochondria are the microscopic cellular combustion engines that utilize glucose and fat to provide our cells with ATP. Given its role in locomotion, skeletal muscle of the lower extremities is abundant with mitochondria. Mitochondrial oxidation of fuel substrates (glucose, fatty acids) converts to acetyl-CoA. The subsequent condensation of acetyl-CoA with oxaloacetate within the mitochondrial matrix forms citrate, initiating the tricarboxylic acid cycle (TCA). Generation of TCA cycle intermediates results in the reduction of electron carriers (NADH and $\mathrm{FADH}_{2}$ ), which in turn shuttle electrons to the electron transport chain of the inner mitochondrial membrane. Electron transfer results in the translocation of protons across the inner mitochondrial membrane, resulting in electro-chemical potential. This membrane potential is used by ATP synthase to phosphorylate adenosine diphosphate (ADP; i.e., oxidative phosphorylation; Mitchell, 1961). Critical to the process of oxidative phosphorylation is the requirement for a terminal electron acceptor, a role fulfilled in mitochondria by molecular $\mathrm{O}_{2}$. Thus, a patent arterial circulation is critical in providing cells and in particular their mitochondria with $\mathrm{O}_{2}$ to support mitochondrial respiration and thus oxidative phosphorylation (Figure 1).

As described above, the pathophysiology of the limb manifestations in lower extremity PAD is complex, and extends beyond the atherosclerotic occlusive disease and inadequate $\mathrm{O}_{2}$ delivery to the mitochondria of the working muscles. Since more than $90 \%$ of $\mathrm{O}_{2}$ is consumed within mitochondria (Rolfe and Brown, 1997), deficits in mitochondrial respiratory capacity and/or function may also contribute to limb manifestations in PAD patients. Early studies suggest incomplete oxidation of fuel substrates in PAD skeletal muscle biopsies as indicated by accumulation of metabolic intermediates (Brass and Hiatt, 2000; Pipinos et al., 2007; Hiatt et al., 2015). PAD gastrocnemius muscle displayed accumulation of acylcarnitines, which is suggestive of incomplete acyl-CoAs oxidation. Additionally, short-chain acylcarnitine accumulation was associated with impaired peak exercise performance (Hiatt et al., 1992). Muscle lactate concentrations have also been reported to be elevated in PAD patients, reflecting anaerobic glucose oxidation (Hiatt et al., 1992). While incomplete oxidation of substrates might be reflective of reduced mitochondrial oxidative capacity, it may also reflect adaptations in muscle that are independent of mitochondrial function, namely reduced blood flow.

\section{ASSESSMENT METHODS FOR SKELETAL MUSCLE MITOCHONDRIAL RESPIRATORY CAPACITY AND FUNCTION}

The numerous analytical techniques and read-outs of mitochondrial function can often lead to confusion as to the role of the mitochondrion in a given pathology. Thus, careful consideration in selecting appropriate analytical endpoints and interpretation of the data that these techniques provide 


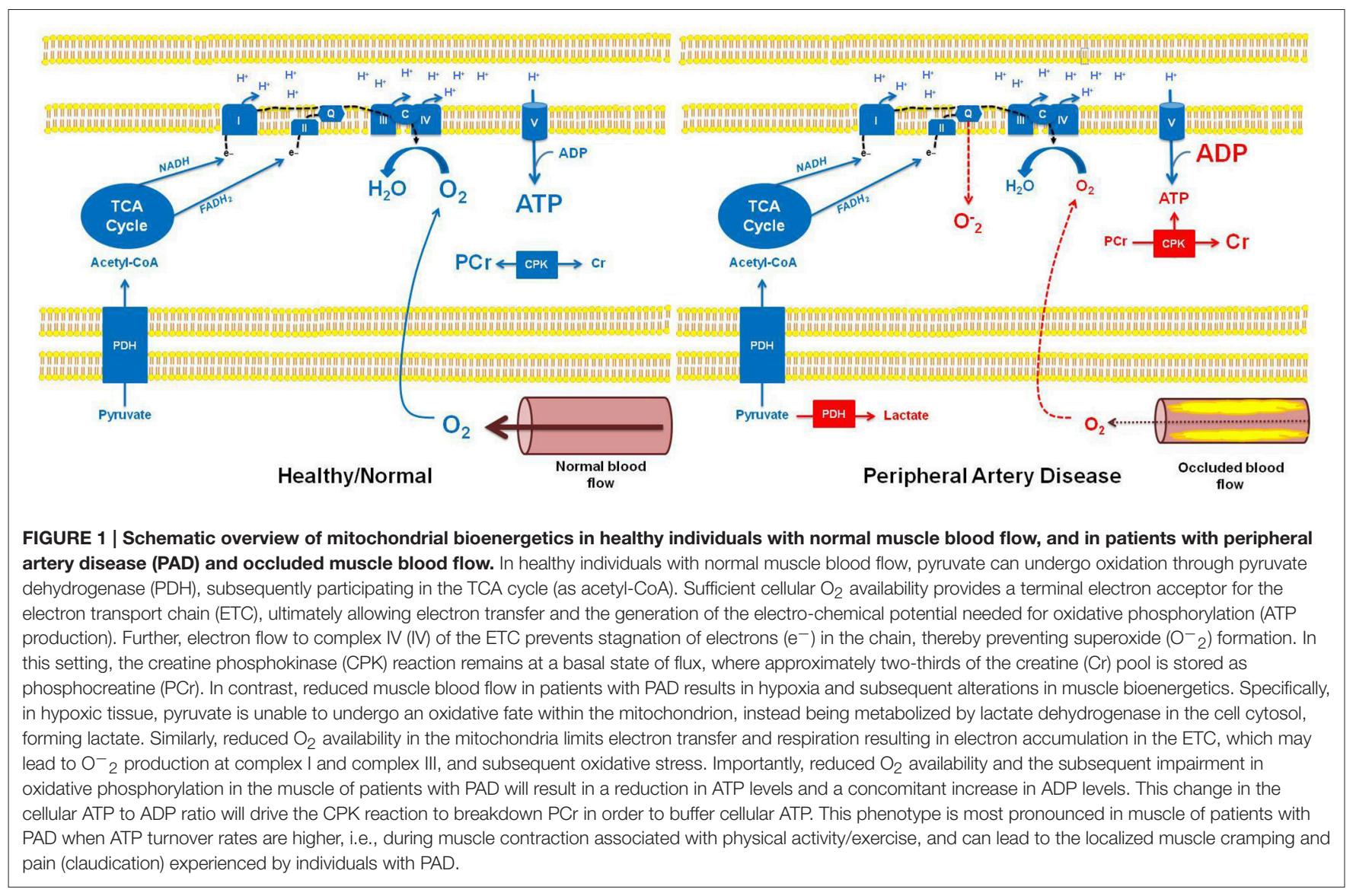

is important if robust conclusions are to be made. Available techniques for measuring mitochondrial function (Figure 2) can be broadly categorized into: (i) in vivo measures of tissue oxidative capacity, (ii) in vitro determination of mitochondrial respiration in tissue samples or isolated mitochondria preparations, assaying ATP production rates, membrane potential, or ROS production in isolated organelles, or the quantification of mitochondrial protein abundance/enzyme activity. The pros and cons of these approaches are discussed in brief below.

Mitochondrial oxidative capacity can be assessed in vivo by ${ }^{31}$ Phosphorus Magnetic Resonance Spectroscopy ( ${ }^{31}$ PMRS; Hoult et al., 1974; Ackerman et al., 1980), a non-invasive method for the determination of relative concentrations of high-energy phosphate metabolites, principally phosphocreatine (PCr), and their kinetic changes during exercise (or ischemia) and their subsequent recovery rate constants. At the onset of intense exercise, $\mathrm{PCr}$ supplies ADP with its high-energy phosphate to form ATP, thereby buffering intracellular ATP levels, resulting in $\mathrm{PCr}$ degradation; the mean rate of $\mathrm{PCr}$ degradation during exercise provides an estimate of the failure of oxidative (plus glycolytic) ATP synthesis to meet ATP demand/turnover (Kemp et al., 1995b, 2001), with the initial rate of the PCr degradation being a measure of ATP turnover rate. During recovery following exercise, $\mathrm{PCr}$ and ADP return to baseline concentrations; the initial rate of PCr re-synthesis post-exercise serves as an estimate of the end-exercise rate of oxidative ATP synthesis (Blei et al., 1993).

Tissue oximetry by near-infrared spectroscopy (NIRS) offers a non-invasive approach for the assessment of the kinetics of (muscle) tissue $\mathrm{O}_{2}$ saturation and hemoglobin/deoxyhemoglobin levels (Jobsis, 1977). Analogous to PCr kinetics determined by ${ }^{31} \mathrm{PMRS}$, the rate of $\mathrm{O}_{2}$ desaturation in muscle during exercise reflects the rate of failure of $\mathrm{O}_{2}$ delivery to meet tissue $\mathrm{O}_{2}$ demand, and the post-exercise/occlusion recovery of $\mathrm{O}_{2}$ saturation rate correlates well with $\mathrm{PCr}$ recovery rate and provides an index of muscle respiratory capacity (Kemp et al., 2001; Nagasawa et al., 2003; Ryan et al., 2013).

Determination of muscle oxidative capacity in vivo offers several advantages. Firstly, oxidative capacity is determined under physiologic conditions. Further, measurements can be made non-invasively. While determination of PCr recovery can be costly, NIRS offers a more affordable alternative to highly costly MRI scanners, which correlates well with MRI-based approaches (Ryan et al., 2013) and muscle biopsy assessment of muscle respiratory capacity (Ryan et al., 2014). However, a caveat of these approaches is that while the capacity for oxidative phosphorylation can be quantified in vivo, it is difficult to discern whether a deficit in oxidative capacity results from impaired blood flow, reduced mitochondrial volume 


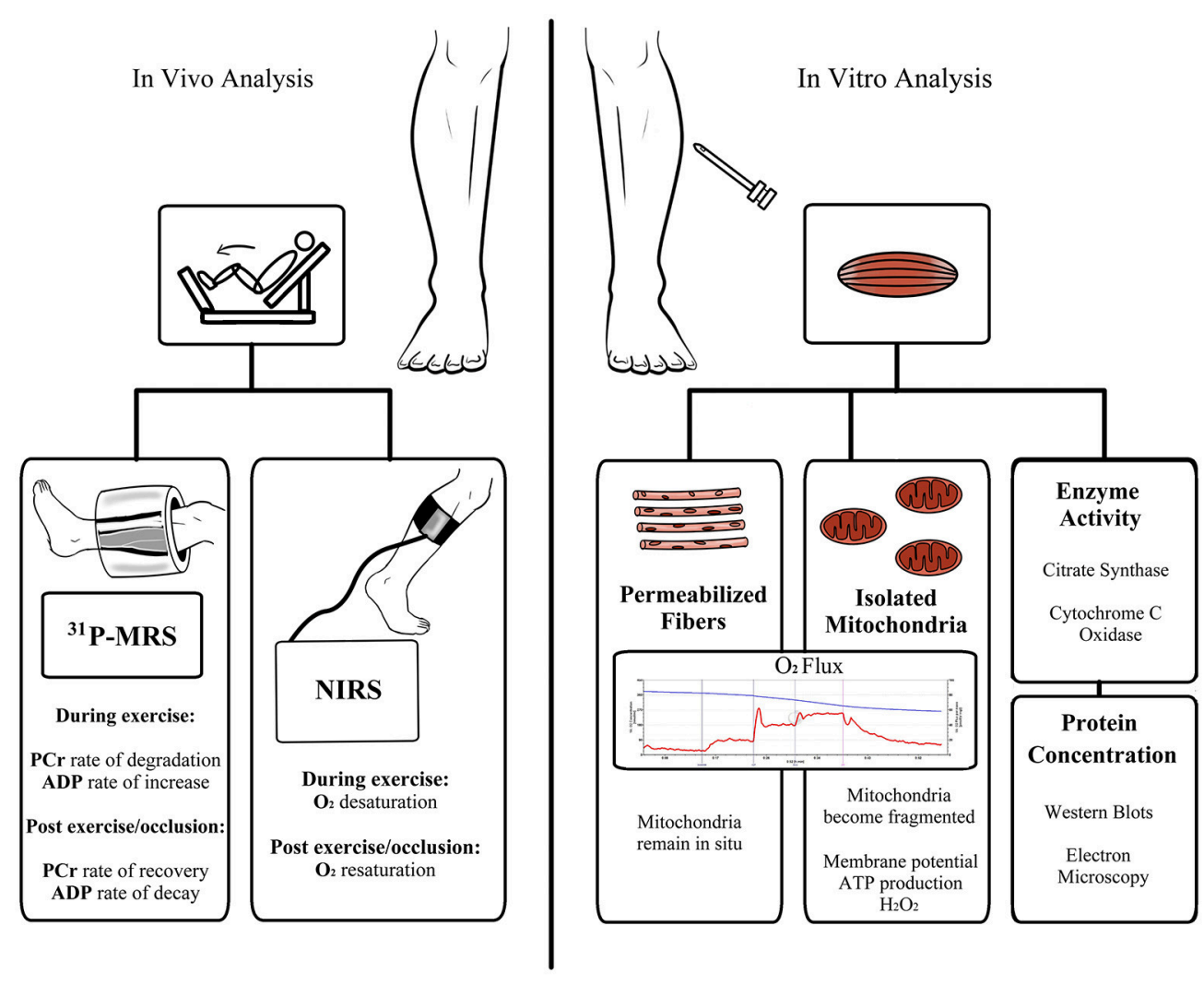

FIGURE 2 | Schematic overview of available methods for the assessment of skeletal muscle mitochondrial respiratory capacity and function.

Abbreviations: ADP, adenosine diphosphate; ATP, adenosine triphosphate; $\mathrm{H}_{2} \mathrm{O}_{2}$, hydrogen peroxide; NIRS, near-infrared spectroscopy; PCr, phosphocreatine;

${ }^{31}$ PMRS, ${ }^{31}$ Phosphorus magnetic resonance spectroscopy.

density, altered mitochondria quality, or a combination of these factors.

Biochemical analysis of muscle biopsy samples allows mitochondrial function to be assayed in tightly controlled systems. Typically, mitochondria can be isolated from muscle tissue by homogenization and centrifugation, or muscle fiber bundles can be chemically permeabilized and mitochondria studied in situ. For several decades, studying respiration or ATP production in isolated organelles was the gold-standard approach. However, mechanical isolation of mitochondria from muscle tissue liberates only $\sim 50 \%$ of the total mitochondrial pool (Rasmussen et al., 2003). Further, more recent evidence has highlighted that the reticular structure and network formed by mitochondria in situ is significantly disrupted by isolation from skeletal muscle (Picard et al., 2011a,b). Importantly, this may lead to erroneous data concerning mitochondrial functionality (Picard et al., 2010). This may also explain the contradictory findings between mitochondrial respiratory capacity in isolated mitochondria and in muscle fiber bundles from PAD patients discussed in the following section. The development of high-resolution respirometry methodologies where mitochondrial respiration can be determined in permeabilized fiber preparations (Saks et al., 1998) now allows mitochondrial function to be determined when organelles remain in their normal architectural environment. In addition, the entire mitochondrial pool can be studied in $\sim 5 \mathrm{mg}$ (wet weight) of tissue, whereas much greater tissue volumes are typically required to isolate a viable pool of mitochondria. Biochemical approaches, such as high-resolution respirometry, allow for the determination of mitochondrial respiratory capacity and function in skeletal muscle samples (Kuznetsov et al., 2008; Gnaiger, 2009). Further, the function of specific complexes of the electron transport system can be determined. Collectively, such biochemical approaches allow detailed information to be generated on mitochondrial function. However, it should be noted that in vitro approaches typically employ supraphysiological $\mathrm{O}_{2}$ tensions and substrate concentrations. Clearly, these caveats need to be considered when interpreting data.

Finally, since assaying mitochondrial function in biopsy samples requires the use of specialized equipment and needs to be performed on fresh tissue, investigators often assay the protein levels and/or activity of mitochondrial enzymes spectrophotometrically as indicators of muscle oxidative function/capacity. These measurements can serve as valid surrogate markers of mitochondrial volume density and thus oxidative capacity. In particular, cardiolipin content and citrate synthase activity correlate well with electron microscopic determination of mitochondrial volume density in healthy adults 
(Larsen et al., 2012). Moreover, cytochrome C oxidase activity correlates with mitochondrial respiratory capacity (Larsen et al., 2012), at least in young healthy adults.

The various approaches described above allow different parameters of muscle mitochondrial performance to be determined, each having its own strengths and weaknesses. Due to the complementary nature of in vivo and in vitro measurements, a combination of approaches within a single study will likely offer the most comprehensive evaluation of skeletal muscle mitochondrial function.

\section{SKELETAL MUSCLE MITOCHONDRIAL FUNCTION IN PAD}

\section{Evidence of Altered Muscle Oxidative Capacity in vivo}

A number of descriptive studies in small cohorts of PAD patients, primarily claudicants, have determined skeletal muscle oxidative capacity in PAD by means of ${ }^{31}$ PMRS or NIRS. The majority of these studies demonstrate increased PCr hydrolysis during exercise, and slower $\mathrm{PCr}$ recovery post-exercise in patients with PAD (Keller et al., 1985; Hands et al., 1986; Zatina et al., 1986; Wahl et al., 1994; Kemp et al., 1995a, 2001; Di Marzo et al., 1999; Pipinos et al., 2000; Greiner et al., 2006; Anderson et al., 2009). Greater PCr degradation during submaximal exercise reflects a greater mismatch between oxidative phosphorylation and ATP turnover. Slower PCr recovery post-exercise indicates reduced capacity for mitochondrial ATP production in PAD. To account for a confounding effect of exercise-induced ischemia and $\mathrm{pH}$ change (Kemp, 2004; Pipinos et al., 2007), PCr recovery was evaluated following mild isometric exercise by Pipinos et al. (2000) in order to minimize $\mathrm{pH}$ changes, which showed a similar slower PCr recovery in PAD patients compared to control, again suggesting reduced skeletal muscle oxidative capacity in patients with PAD. Collectively, these studies demonstrate that skeletal muscle oxidative capacity is diminished in PAD, where there is a greater reliance in substrate level phosphorylation to support cellular ATP demand. Additionally, prolonged PCr recovery was associated with poor treadmill performance but not with calf muscle perfusion in a cross-sectional study of 85 patients with mild to moderate PAD (mean ABI, 0.69), suggesting a role for reduced muscle oxidative capacity in the functional impairment in PAD, possibly independent of reduced blood flow (Anderson et al., 2009).

In support of PCr kinetic data, measurement of $\mathrm{O}_{2}$ kinetics by NIRS suggest increased muscle deoxygenation during exercise and slower reoxygenation post-exercise in PAD (Kooijman et al., 1997; Kemp et al., 2001; Egun et al., 2002; Comerota et al., 2003), where $\mathrm{O}_{2}$ recovery rate correlate well with $\mathrm{PCr}$ recovery rate in PAD patients (Kemp et al., 2001). Collectively, both MRS and NIRS provide robust evidence in vivo of impaired oxidative capacity in skeletal muscle of PAD patients. However, given the nature of these measurements, it is not possible to discern the underlying cause of reduced skeletal muscle oxidative capacity in PAD. For example, whether reduced oxidative capacity is the result of reduced mitochondrial volume density and/or diminished mitochondrial quality cannot be concluded from these in vivo measurements. Determination of mitochondrial function and protein abundance in biopsy samples is required to delineate potential deficits in mitochondrial density and/or quality in the metabolic myopathy that accompanies PAD.

\section{Evidence of Altered Mitochondrial Function in Skeletal Muscle of PAD Patients}

A number of studies have attempted to determine skeletal muscle mitochondrial function in PAD patients (Jansson et al., 1988; Pipinos et al., 2003, 2006; Koutakis et al., 2015; van Schaardenburgh et al., 2016). In a series of studies, Pipinos and colleagues (Pipinos et al., 2003, 2006; Koutakis et al., 2015) evaluated mitochondrial respiratory capacity in saponinpermeabilized myofiber bundles from the gastrocnemius muscle of PAD patients using Clark-type oxygen electrodes. In the first of these studies, mitochondrial respiration was determined in skeletal muscle from 9 patients with advanced PAD (mean ABI, 0.4) and 9 PAD-free individuals (Pipinos et al., 2003). These authors found that ADP stimulated respiration supported by complex I was lower in PAD patients compared to controls, suggesting a quantitative deficit in mitochondrial respiratory capacity in PAD. Further, mitochondrial coupling control determined after the inhibition of adenine nucleotide translocase (via atractyloside titration) was lower in PAD vs. PAD-free individuals (Pipinos et al., 2003), suggesting altered mitochondrial quality (i.e., coupling control) in skeletal muscle of PAD patients.

In a consecutive study, Pipinos et al. (2006) combined respirometric and spectrophotometric measurements in gastrocnemius muscle biopsies of 25 advanced PAD (mean ABI, 0.34 ) and 16 PAD-free individuals to assess several parameters of muscle oxidative capacity. Mitochondrial respiration supported by electron transfer from complex I and complex II of the electron transport chain was assayed. Further, titration of mitochondrial inhibitors and specific electron donors was used to assay the respiratory capacity of complex III and complex IV of the electron transport chain. When normalized to citrate synthase activity, the investigators reported lower ADP-stimulated respiration supported by complex I in PAD patients, but no differences between groups in respiration supported by complex II, suggesting substrate specific deficits in respiratory capacity of muscle mitochondria of PAD patients. Moreover, these authors reported lower respiratory capacity at the level of complex III and complex IV, which likely reflect an overall reduction in mitochondrial respiratory capacity in muscle of PAD patients. In support of these respirometric data, spectrophotometric assays of mitochondrial enzyme activities normalized to citrate synthase activity demonstrated lower $\mathrm{NADH}$ dehydrogenase (complex I), ubiquinol cytochrome $\mathrm{c}$ oxidoreductase (complex III) and cytochrome c oxidoreductase (complex IV) activities in muscle of PAD patients, where complex I and III enzyme activities correlated with complex-I-supported respiration and complex-III-supported respiration, respectively (Pipinos et al., 2006). In agreement with the above findings, Brass et al. (2001) demonstrated lower NADH dehydrogenase activity 
when normalized to citrate synthase activity in gastrocnemius muscle biopsies from 17 PAD (mean ABI, 0.64) patients compared with 9 controls. However, these authors found no significant differences in mitochondrial enzyme activities of other electron transport chain complexes. These contrasting results may relate to disparate control groups and/or severity of PAD between studies (advanced vs. moderate ABI values).

More recently, the group led by Pipinos (Koutakis et al., 2015) provided further evidence of skeletal muscle mitochondrial dysfunction in PAD. Studying $30 \mathrm{PAD}$ (mean ABI, 0.55) and $30 \mathrm{PAD}$-free patients, the authors reported significantly lower ADP-stimulated respiration supported by complex I and complex IV-dependent respiration normalized to citrate synthase activity in PAD patients vs. controls (Koutakis et al., 2015). In contrast to their previous report (Pipinos et al., 2006), PAD patients did not have diminished complex III-dependent respiration. Once again, this possibly pertains to differences in the characteristics of the control groups between studies, with the control group being closely matched to the PAD group in the latest study (Koutakis et al., 2015) compared with a relatively healthier control group in the prior study (Pipinos et al., 2006). Furthermore, fluorescent microscopy data revealed an irregular, uneven and patchy distribution of mitochondria in PAD gastrocnemius myofibers, with absence of mitochondria in desmin-dense myofiber areas that correlated with decreased complex I- and IV-dependent respiration. In addition, this group has reported increased protein carbonyl and 4-hydroxy-2-nonenal (4-HNE) contents in gastrocnemius myofibers in PAD patients compared to PAD-free individuals, across all myofiber types (Pipinos et al., 2006; Weiss et al., 2013; Koutakis et al., 2014), indicating elevated oxidative stress in muscle of patients with PAD. Increased oxidative damage in muscle samples from these PAD patients was associated with reduced myofiber size, and clinical disease progression (Weiss et al., 2013; Koutakis et al., 2014). Type II (fast-twitch) and I/II fibers (mixed) myofibers had higher carbonyl content (i.e., greater oxidative damage) and displayed a greater reduction in size compared to Type I fibers (slow-twitch) in PAD vs. PAD-free gastrocnemius muscle. The oxidative damage and reduced fiber size is also coupled to a shift from Type II myofibers to Type I and Type I/II fibers (Koutakis et al., 2014). These findings suggest that oxidative stress and changes in the mitochondrial structure and architecture may contribute to the lower respiratory capacity of skeletal muscle from PAD patients. Furthermore, data from a recent study by White et al. (2016) is suggestive of impaired clearance of damaged mitochondria despite greater activation of mitophagy in gastrocnemius muscle biopsies from PAD patients, which may influence mitochondrial turnover rates.

Besides the observations in patients, evidence from studies using a mouse model of hindlimb ischemia indicates that disruption of mitochondrial detoxifying/antioxidant systems may partially account for compromised mitochondrial respiration and skeletal muscle abnormalities in PAD patients. In these studies, mitigation of mitochondrial oxidative stress by mitochondrial-targeted therapy improved mitochondrial function (Ryan et al., 2016a,b). In addition, mice deficient in mitochondrial aldehyde dehydrogenase 2 , an enzyme responsible for toxic aldehyde clearance (such as 4-HNE), exhibited greater gastrocnemius muscle atrophy in response to chronic hindlimb ischemia compared to wild-type mice (Liu et al., 2015), further supporting a role for oxidative stress in the mitochondrial myopathy accompanying PAD.

It should be noted that while measurement of citrate synthase activity correlates well with mitochondrial volume density in healthy adults (Larsen et al., 2012), whether this is true in PAD patients has not been confirmed. Several studies suggest no significant differences in mitochondrial volume density as indicated by citrate synthase activity in the gastrocnemius muscle between PAD and PAD-free patients (Bhat et al., 1999; Wang et al., 1999; Brass et al., 2001; Hou et al., 2002; Pipinos et al., 2006). However, other studies report higher citrate synthase activity in gastrocnemius muscle of the claudicant leg than the asymptomatic leg in patients with unilateral PAD or between legs of different PAD severity (Jansson et al., 1988; Hiatt et al., 1996), in line with mitochondrial volume density data determined by electron microscopy (EM) in anterior tibial muscle of unilateral PAD patients (Angquist and Sjöström, 1980). Yet, a more recent study has demonstrated reduced mitochondrial volume density by EM in the vastus lateralis of 14 claudicants (mean ABI, 0.73; Baum et al., 2016). Differences in muscle sampling sites, severity of PAD, or methodologies used may account for the disparate findings between studies. Non etheless, muscle citrate synthase activity was recently identified as a predictor of mortality rate in PAD patients, with mid-range values being associated with greater survival (Thompson et al., 2015).

While the current literature is somewhat conflicting, the above data suggest reduced muscle mitochondrial respiratory capacity in PAD, which is likely accompanied by a change in the mitochondrial volume density, at least in the more severe manifestations of the disease. However, there is a paucity of data on skeletal muscle mitochondrial coupling and flux control in patients with PAD. Early work by Elander et al. (1985) studied both mitochondrial respiratory capacity and coupling control in isolated mitochondrial sub-populations from the gastrocnemius muscle of PAD patients (mean ABI, 0.58 ) and PAD-free controls. Contrary to the more recent evidence suggesting impaired mitochondrial respiration, these authors reported higher respiration supported by complex I and respiratory control ratio for complex I and II in specific isolated mitochondrial sub-populations of PAD vs. PAD-free patients. The discrepant findings may reflect certain limitations with studying mitochondrial respiration in isolated mitochondria than in permeabilized muscle fibers, such as low yields and disruption of the mitochondrial network (Picard et al., 2010, 2011a,b). Indeed, van Schaardenburgh et al. (2016) recently studied both mitochondrial respiratory capacity and coupling control in permeabilized muscle fibers from the gastrocnemius muscle of 11 patients with PAD (mean ABI, 0.65) and 11 PAD-free healthy older adults. They found lower mitochondrial respiration supported by complex I but normal complex-IIsupported respiration in PAD vs. PAD-free patients, in contrast to the previous findings of Elander et al. (1985). However, when 
normalized to citrate synthase activity, mitochondrial respiration ceased to differ between PAD and PAD-free patients, even though citrate synthase activity values per se did not differ between groups. Yet, the coupling control efficiency (similar to respiratory control ratio) for complex I was 2.5 times lower in PAD vs. healthy older adults, which may serve as an index of intrinsic mitochondrial dysfunction at complex I. Moreover, the coupling control factor (and index of respiratory control) for complex II was greater in PAD patients vs. controls, perhaps suggesting a compensatory response to impaired complex I function. Here, it should be noted that this study (van Schaardenburgh et al., 2016) was designed to examine the acute effects of exercise on mitochondrial respiration within groups separately, possibly explaining why the groups were not agematched, and thus, any between group baseline comparisons should be interpreted with caution. Yet, these recent findings (van Schaardenburgh et al., 2016) provide valuable insight in mitochondrial quality and offer the basis for future studies to comprehensively characterize skeletal muscle mitochondrial function in PAD.

\section{Existing Therapeutic Approaches to Restore Mitochondrial Function and Skeletal Muscle Abnormalities in PAD}

Exercise training, in particular supervised exercise therapy, is recommended for PAD (Fokkenrood et al., 2013; Rooke et al., 2013), for its beneficial effects on functional capacity (McDermott et al., 2009). A limited number of studies in patients with $\mathrm{PAD}$ have highlighted the potential of exercise therapy to improve skeletal muscle metabolism and mitochondrial function. In $10 \mathrm{PAD}$ patients, improved exercise performance with supervised exercise training was associated with improved lipid oxidation (as indicated by altered carnitine metabolism; Hiatt et al., 1990, 1996). Supervised exercise training resulted in an increase in pyruvate- and L-malateinduced mitochondrial respiration of calf muscle from 8 PAD patients compared to 7 untrained patients and 11 healthy controls as demonstrated by in vitro approaches (Hou et al., 2002). Furthermore, a pilot study has suggested improved $\mathrm{PCr}$ recovery kinetics indicating enhanced oxidative capacity in $\mathrm{PAD}$ patients following supervised exercise training, but no comparisons were made to a control group (Brizendine et al., 2014). Pentoxifylline, an FDA approved vasoactive drug (phosphodiesterase inhibitor) for intermittent claudication, has been shown to improve oxidative capacity assessed by $\mathrm{PCr}$ recovery kinetics, which in turn is associated with improved functional capacity in patients with PAD (Pipinos et al., 2002).

A limited number of studies have also investigated the effects of revascularization procedures on mitochondrial function with inconsistent results between studies. Improved but not complete restoration of oxidative capacity determined by PCr recovery kinetics in lower extremity muscles has been demonstrated in PAD patients who underwent lower extremity revascularization procedures (Schunk et al., 1998; West et al., 2012). In a third study, treatment of PAD patients with lower limb PTA or bypass surgery failed to demonstrate significant improvement in oxidative metabolism determined by $\mathrm{PCr}$ recovery kinetics in calf muscle, albeit normalization of hemodynamic parameters (Zatina et al., 1986). Collectively, clear data on the role of surgical revascularization in improving skeletal muscle oxidative capacity in PAD patients is lacking. In contrast, the majority of data on exercise training suggests efficacy in terms of restoring muscle oxidative capacity in PAD patients. Thus, one may conclude that reduced muscle mitochondrial function in PAD may be influenced more directly by reduced muscle contraction, underscoring the importance of physical activity and exercise training strategies in the management of PAD. Since oxidative stress may be responsible for some of the deficits in muscle mitochondrial function observed in patients with $\mathrm{PAD}$, therapies targeting mitochondrial antioxidant systems may hold therapeutic value. Indeed, targeted antioxidant therapy restores mitochondrial function in a rodent model of hindlimb ischemia (Ryan et al., $2016 a, b)$. The therapeutic value of manipulating mitochondrial antioxidant systems as well as other mitochondrial quality control mechanisms, including mitochondrial dynamics (fusionfission balance), and mitochondrial turnover (mitophagy) in the context of PAD has been comprehensively reviewed elsewhere (Ueta et al., 2017).

Although this review focuses on the role of mitochondria in the myopathy of lower extremities PAD, additional factors also contribute to the development of PAD. Frequently, these processes are the result of an orchestrated response to ischemia and involve numerous cell types. In the gastrocnemius muscle of PAD patients, vascular smooth muscle cells have been found to shift to a pro-fibrotic phenotype, expressing transforming growth factor-beta 1 (TGF- $\beta 1$; a pro-fibrotic cytokine; Ha et al., 2016). Elevated TGF- $\beta 1$ expression was associated with accumulation of fibroblasts and collagen deposition in the muscle biopsies from these PAD patients. As PAD progresses, collagen deposition expands from the perivascular area and infiltrates the gastrocnemius myofibers (Ha et al., 2016). Further, in the ischemic microenvironment, endothelial and skeletal muscle cells produce angiogenic factors, such as vascular endothelial growth factor (VEGF) and angiopoietin1. In vitro data suggest that VEGF and angiopoietin-1 secreted by skeletal muscle and endothelial cells in response to ischemia may play a role in muscle remodeling (McClung et al., 2015). In addition, rodents with hindlimb ischemia show improved muscle regeneration when treated with angiogenic and myogenic growth factors (Borselli et al., 2010). Hence, targeting the multicellular skeletal muscle environment may be of therapeutic value in the management of the myopathy associated with PAD.

\section{Summary and Future Directions}

Collectively, current evidence from in vivo and in vitro methodologies suggests reduced skeletal muscle oxidative capacity in PAD. Diminished skeletal muscle oxidative capacity appears to result from both impaired blood flow and altered mitochondrial respiratory capacity and quality. Although these findings are important, they are the product 
of a number of small and sometimes disparate exploratory studies, which do not allow for firm conclusions to be drawn regarding the role of mitochondrial function in PAD. The tight coupling between the vasculature, oxygen delivery, and muscle mitochondrial respiration constitutes a dynamic system of complex interactions that may be best explored concurrently with a combination of in vivo and in vitro methodologies. In particular, we suggest future studies to aim to: (i) be sufficiently-powered; (ii) use appropriate controls to draw comparisons to by matching PAD and control groups on potential confounders, such as age, gender, weight, smoking status, coronary artery disease, diabetes, dyslipidemia, and hypertension; (iii) stratify PAD based on severity/clinical manifestations (ie., asymptomatic, claudication, atypical, critical limb ischemia); (iv) integrate a combination of methodologies in order to assess oxidative capacity in vivo, determine intrinsic mitochondrial respiratory capacity, and coupling control ratios (mitochondrial quality), and muscle mitochondrial volume density in muscle biopsies of the same patients; (v) determine the relationships between mitochondrial function, clinical PAD parameters, and functional limitations which was not feasible in the majority of prior studies due to being relatively underpowered. Inclusion of patients with unilateral PAD and assessments on both limbs would also be highly informative. Moreover, analysis of mitochondrial function in biopsies collected from different regions of the affected muscle in the same patient will provide additional important information on the nature of mitochondrial dysfunction in muscle from PAD patients. Furthermore, data on the longterm effects of revascularization procedures on mitochondrial function is currently lacking. Finally, transcriptome-wide analysis of muscle from patients with PAD would be useful and may identify new therapeutic targets worthy of further investigations.

\section{REFERENCES}

Aboyans, V., Criqui, M. H., Abraham, P., Allison, M. A., Creager, M. A., Diehm, C., et al. (2012). Measurement and interpretation of the ankle-brachial index: a scientific statement from the American Heart Association. Circulation 126, 2890-2909. doi: 10.1161/CIR.0b013e318276fbcb

Ackerman, J. J., Grove, T. H., Wong, G. G., Gadian, D. G., and Radda, G. K. (1980). Mapping of metabolites in whole animals by 31P NMR using surface coils. Nature 283, 167-170. doi: 10.1038/283167a0

Allison, M. A., Ho, E., Denenberg, J. O., Langer, R. D., Newman, A. B., Fabsitz, R. R., et al. (2007). Ethnic-specific prevalence of peripheral arterial disease in the United States. Am. J. Prev. Med. 32, 328-333. doi: 10.1016/j.amepre.2006.12.010

Amoh-Tonto, C. A., Malik, A. R., Kondragunta, V., Ali, Z., and Kullo, I. J. (2009). Brachial-ankle pulse wave velocity is associated with walking distance in patients referred for peripheral arterial disease evaluation. Atherosclerosis 206, 173-178. doi: 10.1016/j.atherosclerosis.2009.02.003

Anderson, J. D., Epstein, F. H., Meyer, C. H., Hagspiel, K. D., Wang, H., Berr, S. S., et al. (2009). Multifactorial determinants of functional capacity in peripheral arterial disease: uncoupling of calf muscle perfusion and metabolism. J. Am. Coll. Cardiol. 54, 628-635. doi: 10.1016/j.jacc.2009.01.080

Angquist, K. A., and Sjöström, M. (1980). Intermittent claudication and muscle fiber fine structure: morphometric data on mitochondrial volumes. Ultrastruct. Pathol. 1, 461-470. doi: 10.3109/01913128009140552

\section{CONCLUSION}

The lack of a clear understanding of the role of mitochondrial dysfunction in PAD represents a significant roadblock in the development of novel strategies to restore muscle function, ameliorate limb symptoms, and improve functional capacity in PAD patients. Therefore, generation of new data concerning the role of bioenergetics in PAD may contribute to the development of novel therapies aimed at reducing morbidity in patients living with PAD. Considering the rising prevalence of PAD, the persistence of PAD-associated myopathy after revascularization, its functional and economic impact, and the limited therapeutic options that currently exist, further research in this field is warranted.

\section{AUTHOR CONTRIBUTIONS}

Substantial contribution to the concept and interpretation of available evidence (All authors); Drafted the work (VR and CP). Revising it critically for important intellectual content (ON, GF, $\mathrm{ZC}$, and $\mathrm{BR}$ ). Final approval of the version to be published (All authors). Agreement to be accountable for all aspects of the work in ensuring that questions related to the accuracy or integrity of any part of the work are appropriately investigated and resolved (All authors).

\section{FUNDING}

Seed funding for this project was supplied by the Department of Surgery, UTMB. This work was also supported by the following grants: R56 AG051267, P30 AG024832 and SHC84090 , and was conducted with the support of the Institute for Translational Sciences at UTMB, supported in part by a Clinical and Translational Science Award (UL1TR000071).

Armstrong, D. G., Wrobel, J., and Robbins, J. M. (2007). Guest editorial: are diabetes-related wounds and amputations worse than cancer? Int. Wound J. 4, 286-287. doi: 10.1111/j.1742-481X.2007.00392.x

Baum, O., Torchetti, E., Malik, C., Hoier, B., Walker, M., Walker, P. J., et al. (2016). Capillary ultrastructure and mitochondrial volume density in skeletal muscle in relation to reduced exercise capacity of patients with intermittent claudication. Am. J. Physiol. Regul. Integr. Comp. Physiol. 310, R943-R951. doi: 10.1152/ajpregu.00480.2015

Beckmann, M., Jacomella, V., Kohler, M., Lachat, M., Salem, A., Amann-Vesti, B., et al. (2015). Risk stratification of patients with peripheral arterial disease and abdominal aortic aneurysm using aortic augmentation index. PLoS ONE 10:e0139887. doi: 10.1371/journal.pone.0139887

Bhat, H. K., Hiatt, W. R., Hoppel, C. L., and Brass, E. P. (1999). Skeletal muscle mitochondrial DNA injury in patients with unilateral peripheral arterial disease. Circulation 99, 807-812. doi: 10.1161/01.CIR. 99.6.807

Blei, M. L., Conley, K. E., and Kushmerick, M. J. (1993). Separate measures of ATP utilization and recovery in human skeletal muscle. J. Physiol. 465, 203-222. doi: 10.1113/jphysiol.1993.sp019673

Borselli, C., Storrie, H., Benesch-Lee, F., Shvartsman, D., Cezar, C., Lichtman, J. W., et al. (2010). Functional muscle regeneration with combined delivery of angiogenesis and myogenesis factors. Proc. Natl. Acad. Sci. U.S.A. 107, 3287-3292. doi: 10.1073/pnas.0903875106 
Bragadeesh, T., Sari, I., Pascotto, M., Micari, A., Kaul, S., and Lindner, J. R. (2005). Detection of peripheral vascular stenosis by assessing skeletal muscle flow reserve. J. Am. Coll. Cardiol. 45, 780-785. doi: 10.1016/j.jacc.2004.11.045

Brass, E. P., and Hiatt, W. R. (2000). Acquired skeletal muscle metabolic myopathy in atherosclerotic peripheral arterial disease. Vasc. Med. 5, 55-59. doi: $10.1177 / 1358836$ X0000500109

Brass, E. P., Hiatt, W. R., Gardner, A. W., and Hoppel, C. L. (2001). Decreased NADH dehydrogenase and ubiquinol-cytochrome c oxidoreductase in peripheral arterial disease. Am. J. Physiol. Heart Circ. Physiol. 280, H603-H609. Available online at: http://ajpheart.physiology.org/content/280/2/ H603.long

Brewer, L. C., Chai, H. S., Bailey, K. R., and Kullo, I. J. (2007). Measures of arterial stiffness and wave reflection are associated with walking distance in patients with peripheral arterial disease. Atherosclerosis 191, 384-390. doi: 10.1016/j.atherosclerosis.2006.03.038

Brizendine, J. T., Young, H.-J., McCully, K., and Murrow, J. (2014). Skeletal muscle mitochondrial function and intermittent claudication in patients with peripheral arterial disease following supervised treadmill training. J. Am. Coll. Cardiol. 63:A2074. doi: 10.1016/S0735-1097(14)62077-5

Comerota, A. J., Throm, R. C., Kelly, P., and Jaff, M. (2003). Tissue (muscle) oxygen saturation $\left(\mathrm{StO}_{2}\right)$ : A new measure of symptomatic lower-extremity arterial disease. J. Vasc. Surg. 38, 724-729. doi: 10.1016/S0741-5214(03)01032-2

Coutinho, T., Rooke, T. W., and Kullo, I. J. (2011a). Arterial dysfunction and functional performance in patients with peripheral artery disease: a review. Vasc. Med. 16, 203-211. doi: 10.1177/1358863X11400935

Coutinho, T., Turner, S. T., and Kullo, I. J. (2011b). Aortic pulse wave velocity is associated with measures of subclinical target organ damage. JACC Cardiovasc. Imaging 4, 754-761. doi: 10.1016/j.jcmg.2011.04.011

Diehm, C., Allenberg, J. R., Pittrow, D., Mahn, M., Tepohl, G., Haberl, R. L., et al. (2009). Mortality and vascular morbidity in older adults with asymptomatic versus symptomatic peripheral artery disease. Circulation 120, 2053-2061. doi: 10.1161/CIRCULATIONAHA.109.865600

Di Marzo, L., Miccheli, A., Sapienza, P., Tedesco, M., Mingoli, A., Capuani, G., et al. (1999). 31Phosphorus magnetic resonance spectroscopy to evaluate medical therapy efficacy in peripheral arterial disease. a pilot study. Panminerva Med. $41,283-290$.

Egun, A., Farooq, V., Torella, F., Cowley, R., Thorniley, M. S., and McCollum, C. N. (2002). The severity of muscle ischemia during intermittent claudication. J. Vasc. Surg. 36, 89-93. doi: 10.1067/mva.2002.123678

Elander, A., Sjöström, M., Lundgren, F., Schersten, T., and BylundFellenius, A. C. (1985). Biochemical and morphometric properties of mitochondrial populations in human muscle fibres. Clin. Sci. 69, 153-164. doi: $10.1042 / \mathrm{cs} 0690153$

Fokkenrood, H. J., Bendermacher, B. L., Lauret, G. J., Willigendael, E. M., Prins, M. H., and Teijink, J. A. (2013). Supervised exercise therapy versus non-supervised exercise therapy for intermittent claudication. Cochrane Database Syst. Rev. 23:CD005263. doi: 10.1002/14651858.CD005263.pub3

Fowkes, F. G., Rudan, D., Rudan, I., Aboyans, V., Denenberg, J. O., McDermott, M. M., et al. (2013). Comparison of global estimates of prevalence and risk factors for peripheral artery disease in 2000 and 2010: a systematic review and analysis. Lancet 382, 1329-1340. doi: 10.1016/S0140-6736(13) $61249-0$

Gardner, A. W., and Killewich, L. A. (2001). Lack of functional benefits following infrainguinal bypass in peripheral arterial occlusive disease patients. Vasc. Med. 6, 9-14. doi: 10.1191/135886301668561166

Gerhard-Herman, M. D., Gornik, H. L., Barrett, C., Barshes, N. R., Corriere, M. A., Drachman, D. E., et al. (2016). 2016 AHA/ACC Guideline on the Management of Patients with Lower Extremity Peripheral Artery Disease. A Report of the American College of Cardiology/American Heart Association Task Force on Clinical Practice Guidelines.

Gnaiger, E. (2009). Capacity of oxidative phosphorylation in human skeletal muscle: new perspectives of mitochondrial physiology. Int. J. Biochem. Cell Biol. 41, 1837-1845. doi: 10.1016/j.biocel.2009.03.013

Greiner, A., Esterhammer, R., Messner, H., Biebl, M., Mühlthaler, H., Fraedrich, G., et al. (2006). High-energy phosphate metabolism during incremental calf exercise in patients with unilaterally symptomatic peripheral arterial disease measured by phosphor 31 magnetic resonance spectroscopy. J. Vasc. Surg. 43, 978-986. doi: 10.1016/j.jvs.2006.01.020
Grenon, S. M., Chong, K., Alley, H., Nosova, E., Gasper, W., Hiramoto, J., et al. (2014). Walking disability in patients with peripheral artery disease is associated with arterial endothelial function. J. Vasc. Surg. 59, 1025-1034. doi: 10.1016/j.jvs.2013.10.084

Ha, D. M., Carpenter, L. C., Koutakis, P., Swanson, S. A., Zhu, Z., Hanna, M., et al. (2016). Transforming growth factor-beta 1 produced by vascular smooth muscle cells predicts fibrosis in the gastrocnemius of patients with peripheral artery disease. J. Transl. Med. 14, 39. doi: 10.1186/s12967-016-0790-3

Hands, L. J., Bore, P. J., Galloway, G., Morris, P. J., and Radda, G. K. (1986). Muscle metabolism in patients with peripheral vascular disease investigated by $31 \mathrm{P}$ nuclear magnetic resonance spectroscopy. Clin. Sci. 71, 283-290. doi: $10.1042 / \mathrm{cs} 0710283$

Heinen, Y., Stegemann, E., Sansone, R., Benedens, K., Wagstaff, R., Balzer, J., et al. (2015). Local association between endothelial dysfunction and intimal hyperplasia: relevance in peripheral artery disease. J. Am. Heart Assoc. 4:e001472. doi: 10.1161/JAHA.114.001472

Hiatt, W. R., Armstrong, E. J., Larson, C. J., and Brass, E. P. (2015). Pathogenesis of the limb manifestations and exercise limitations in peripheral artery disease. Circ. Res. 116, 1527-1539. doi: 10.1161/CIRCRESAHA.116.303566

Hiatt, W. R., Nawaz, D., and Brass, E. P. (1987). Carnitine metabolism during exercise in patients with peripheral vascular disease. J. Appl. Physiol. 62, 2383-2387.

Hiatt, W. R., Regensteiner, J. G., Hargarten, M. E., Wolfel, E. E., and Brass, E. P. (1990). Benefit of exercise conditioning for patients with peripheral arterial disease. Circulation 81, 602-609. doi: 10.1161/01.CIR.81.2.602

Hiatt, W. R., Regensteiner, J. G., Wolfel, E. E., Carry, M. R., and Brass, E. P. (1996). Effect of exercise training on skeletal muscle histology and metabolism in peripheral arterial disease. J. Appl. Physiol. 81, 780-788.

Hiatt, W. R., Wolfel, E. E., Regensteiner, J. G., and Brass, E. P. (1992). Skeletal muscle carnitine metabolism in patients with unilateral peripheral arterial disease. J. Appl. Physiol. 73, 346-353.

Hirsch, A. T., Haskal, Z. J., Hertzer, N. R., Bakal, C. W., Creager, M. A., Halperin, J. L., et al. (2006). ACC/AHA 2005 guidelines for the management of patients with peripheral arterial disease (lower extremity, renal, mesenteric, and abdominal aortic): executive summary a collaborative report from the American association for vascular surgery/society for vascular surgery, society for cardiovascular angiography and interventions, society for vascular medicine and biology, society of interventional radiology, and the ACC/AHA task force on practice guidelines (writing committee to develop guidelines for the management of patients with peripheral arterial disease) endorsed by the American association of cardiovascular and pulmonary rehabilitation; national heart, lung, and blood institute; society for vascular nursing; transatlantic intersociety consensus; and vascular disease foundation. J. Am. Coll. Cardiol. 47, 1239-1312. doi: 10.1016/j.jacc.2005.10.009

Hou, X. Y., Green, S., Askew, C. D., Barker, G., Green, A., and Walker, P. J. (2002). Skeletal muscle mitochondrial ATP production rate and walking performance in peripheral arterial disease. Clin. Physiol. Funct. Imaging 22, 226-232. doi: 10.1046/j.1475-097X.2002.00423.X

Hoult, D. I., Busby, S. J., Gadian, D. G., Radda, G. K., Richards, R. E., and Seeley, P. J. (1974). Observation of tissue metabolites using 31P nuclear magnetic resonance. Nature 252, 285-287. doi: 10.1038/252285a0

Jaff, M. R. (2014). Why patients know more about cars than peripheral artery disease. Circulation 130, 1778-1779. doi: 10.1161/CIRCULATIONAHA. 114.012872

Jansson, E., Johansson, J., Sylven, C., and Kaijser, L. (1988). Calf muscle adaptation in intermittent claudication. Side-differences in muscle metabolic characteristics in patients with unilateral arterial disease. Clin. Physiol. 8, 17-29. doi: 10.1111/j.1475-097X.1988.tb00258.x

Jobsis, F. F. (1977). Non-invasive, infrared monitoring of cerebral and myocardial oxygen sufficiency and circulatory parameters. Science 198, 1264-1267. doi: 10.1126/science.929199

Joras, M., and Poredos, P. (2008). The association of acute exercise-induced ischaemia with systemic vasodilator function in patients with peripheral arterial disease. Vasc. Med. 13, 255-262. doi: 10.1177/1358863X08096347

Keller, U., Oberhänsli, R., Huber, P., Widmer, L. K., Aue, W. P., Hassink, R. I., et al. (1985). Phosphocreatine content and intracellular $\mathrm{pH}$ of calf muscle measured by phosphorus NMR spectroscopy in occlusive arterial disease of the legs. Eur. J. Clin. Invest. 15, 382-388. doi: 10.1111/j.1365-2362.1985.tb00289.x 
Kemp, G. J. (2004). Mitochondrial dysfunction in chronic ischemia and peripheral vascular disease. Mitochondrion 4, 629-640. doi: 10.1016/j.mito.2004.07.017

Kemp, G. J., Hands, L. J., Ramaswami, G., Taylor, D. J., Nicolaides, A., Amato, A., et al. (1995a). Calf muscle mitochondrial and glycogenolytic ATP synthesis in patients with claudication due to peripheral vascular disease analysed using 31P magnetic resonance spectroscopy. Clin. Sci. 89, 581-590. doi: $10.1042 /$ cs 0890581

Kemp, G. J., Roberts, N., Bimson, W. E., Bakran, A., Harris, P. L., Gilling-Smith, G. L., et al. (2001). Mitochondrial function and oxygen supply in normal and in chronically ischemic muscle: a combined 31P magnetic resonance spectroscopy and near infrared spectroscopy study in vivo. J. Vasc. Surg. 34, 1103-1110. doi: $10.1067 /$ mva.2001.117152

Kemp, G. J., Thompson, C. H., Taylor, D. J., and Radda, G. K. (1995b). ATP production and mechanical work in exercising skeletal muscle: a theoretical analysis applied to $31 \mathrm{P}$ magnetic resonance spectroscopic studies of dialyzed uremic patients. Magn. Reson. Med. 33, 601-609. doi: 10.1002/mrm. 1910330504

Kooijman, H. M., Hopman, M. T., Colier, W. N., van der Vliet, J. A., and Oeseburg, B. (1997). Near infrared spectroscopy for non-invasive assessment of claudication. J. Surg. Res. 72, 1-7. doi: 10.1006/jsre.1997.5164

Koutakis, P., Miserlis, D., Myers, S. A., Kim, J. K., Zhu, Z., Papoutsi, E., et al. (2015). Abnormal accumulation of desmin in gastrocnemius myofibers of patients with peripheral artery disease: associations with altered myofiber morphology and density, mitochondrial dysfunction and impaired limb function. J. Histochem. Cytochem. 63, 256-269. doi: 10.1369/0022155415569348

Koutakis, P., Weiss, D. J., Miserlis, D., Shostrom, V. K., Papoutsi, E., Ha, D. M., et al. (2014). Oxidative damage in the gastrocnemius of patients with peripheral artery disease is myofiber type selective. Redox Biol. 2, 921-928. doi: 10.1016/j.redox.2014.07.002

Kuznetsov, A. V., Veksler, V., Gellerich, F. N., Saks, V., Margreiter, R., and Kunz, W. S. (2008). Analysis of mitochondrial function in situ in permeabilized muscle fibers, tissues and cells. Nat. Protoc. 3, 965-976. doi: 10.1038/nprot. 2008.61

Larsen, O. A., and Lassen, N. A. (1966). Effect of daily muscular exercise in patients with intermittent claudication. Lancet 2, 1093-1096. doi: 10.1016/ S0140-6736(66)92191-X

Larsen, S., Nielsen, J., Hansen, C. N., Nielsen, L. B., Wibrand, F., Stride, N., et al. (2012). Biomarkers of mitochondrial content in skeletal muscle of healthy young human subjects. J. Physiol. 590, 3349-3360. doi: 10.1113/jphysiol. 2012.230185

Liu, X., Sun, X., Liao, H., Dong, Z., Zhao, J., Zhu, H., et al. (2015). Mitochondrial aldehyde dehydrogenase 2 regulates revascularization in chronic ischemia: potential impact on the development of coronary collateral circulation. Arterioscler. Thromb. Vasc. Biol. 35, 2196-2206. doi: 10.1161/ATVBAHA.115.306012

Mahoney, E. M., Wang, K., Cohen, D. J., Hirsch, A. T., Alberts, M. J., Eagle, K., et al. (2008). One-year costs in patients with a history of or at risk for atherothrombosis in the United States. Circ. Cardiovasc. Qual. Outcomes 1, 38-45. doi: 10.1161/CIRCOUTCOMES.108.775247

Mahoney, E. M., Wang, K., Keo, H. H., Duval, S., Smolderen, K. G., Cohen, D. J., et al. (2010). Vascular hospitalization rates and costs in patients with peripheral artery disease in the United States. Circ. Cardiovasc. Qual. Outcomes 3, 642-651. doi: 10.1161/CIRCOUTCOMES.109.930735

McArdle, W. D., Katch, F. I., and Katch, V. L. (2007). Exercise Physiology: Energy, Nutrition, and Human Performance. Baltimore, MA: Lippincott Williams \& Wilkins.

McClung, J. M., Reinardy, J. L., Mueller, S. B., McCord, T. J., Kontos, C. D., Brown, D. A., et al. (2015). Muscle cell derived angiopoietin-1 contributes to both myogenesis and angiogenesis in the ischemic environment. Front. Physiol. 6:161. doi: $10.3389 /$ fphys.2015.00161

McDermott, M. M., Liu, K., Greenland, P., Guralnik, J. M., Criqui, M. H., Chan, C., et al. (2004). Functional decline in peripheral arterial disease: associations with the ankle brachial index and leg symptoms. JAMA 292, 453-461. doi: 10.1001/jama.292.4.453

McDermott, M. M. (2015). Lower extremity manifestations of peripheral artery disease: the pathophysiologic and functional implications of leg ischemia. Circ. Res. 116, 1540-1550. doi: 10.1161/CIRCRESAHA.114.303517

McDermott, M. M., Ades, P., Guralnik, J. M., Dyer, A., Ferrucci, L., Liu, K., et al. (2009). Treadmill exercise and resistance training in patients with peripheral arterial disease with and without intermittent claudication: a randomized controlled trial. JAMA 301, 165-174. doi: 10.1001/jama.2008.962

McDermott, M. M., Applegate, W. B., Bonds, D. E., Buford, T. W., Church, T., Espeland, M. A., et al. (2013). Ankle brachial index values, leg symptoms, and functional performance among community-dwelling older men and women in the Lifestyle Interventions and Independence for Elders Study. J. Am. Heart Assoc. 2:e000257. doi: 10.1161/JAHA.113.000257

McDermott, M. M., Fried, L., Simonsick, E., Ling, S., and Guralnik, J. M. (2000). Asymptomatic peripheral arterial disease is independently associated with impaired lower extremity functioning: the women's health and aging study. Circulation 101, 1007-1012. doi: 10.1161/01.CIR.101.9.1007

McDermott, M. M., Greenland, P., Ferrucci, L., Criqui, M. H., Liu, K., Sharma, L., et al. (2002). Lower extremity performance is associated with daily life physical activity in individuals with and without peripheral arterial disease. J. Am. Geriatr. Soc. 50, 247-255. doi: 10.1046/j.1532-5415.2002.50055.x

McDermott, M. M., Guralnik, J. M., Ferrucci, L., Tian, L., Liu, K., Liao, Y., et al. (2008). Asymptomatic peripheral arterial disease is associated with more adverse lower extremity characteristics than intermittent claudication. Circulation 117, 2484-2491. doi: 10.1161/CIRCULATIONAHA.107.736108

Mitchell, P. (1961). Coupling of phosphorylation to electron and hydrogen transfer by a chemi-osmotic type of mechanism. Nature 191, 144-148. doi: $10.1038 / 191144 \mathrm{a} 0$

Mitchell, R. G., Duscha, B. D., Robbins, J. L., Redfern, S. I., Chung, J., Bensimhon, D. R., et al. (2007). Increased levels of apoptosis in gastrocnemius skeletal muscle in patients with peripheral arterial disease. Vasc. Med. 12, 285-290. doi: 10.1177/1358863X07084858

Mozaffarian, D., Benjamin, E. J., Go, A. S., Arnett, D. K., Blaha, M. J., Cushman, M., et al. (2015). Heart disease and stroke statistics-2016 update: a report from the American Heart Association. Circulation. 133, e38-e360. doi: 10.1161/CIR.0000000000000350

Murphy, E., and Steenbergen, C. (2008). Mechanisms underlying acute protection from cardiac ischemia-reperfusion injury. Physiol. Rev. 88, 581-609. doi: 10.1152/physrev.00024.2007

Nagasawa, T., Hamaoka, T., Sako, T., Murakami, M., Kime, R., Homma, T., et al. (2003). A practical indicator of muscle oxidative capacity determined by recovery of muscle $\mathrm{O}_{2}$ consumption using NIR spectroscopy. Eur. J. Sport Sci. 3, 1-10. doi: 10.1080/17461390300073207

Nardi Gomes, T. J., Martins De Albuquerque, I., De Moraes Costa, P., Cardoso, D. M., De Moraes Costa, G., and Da Costa Vieira, J. L. (2015). Association between the ankle-brachial index, intermittent claudication, and physical activity level: what is the influence on the functional capacity of patients with or at high risk of cardiovascular disease? Int. J. Gen. Med. 8, 55-62. doi: 10.2147/IJGM.S76446

Pernow, B., and Zetterquist, S. (1968). Metabolic evaluation of the leg blood flow in claudicating patients with arterial obstructions at different levels. Scand. J. Clin. Lab. Invest. 21, 277-287. doi: 10.3109/00365516809076995

Picard, M., Ritchie, D., Wright, K. J., Romestaing, C., Thomas, M. M., Rowan, S. L., et al. (2010). Mitochondrial functional impairment with aging is exaggerated in isolated mitochondria compared to permeabilized myofibers. Aging Cell 9, 1032-1046. doi: 10.1111/j.1474-9726.2010.00628.x

Picard, M., Taivassalo, T., Gouspillou, G., and Hepple, R. T. (2011a). Mitochondria: isolation, structure and function. J. Physiol. 589, 4413-4421. doi: 10.1113/ jphysiol.2011.212712

Picard, M., Taivassalo, T., Ritchie, D., Wright, K. J., Thomas, M. M., Romestaing, C., et al. (2011b). Mitochondrial structure and function are disrupted by standard isolation methods. PLoS ONE 6:e18317. doi: 10.1371/journal. pone.0018317

Pipinos, I. I, Boska, M. D., Shepard, A. D., Anagnostopoulos, P. V., and Katsamouris, A. (2002). Pentoxifylline reverses oxidative mitochondrial defect in claudicating skeletal muscle. J. Surg. Res. 102, 126-132. doi: 10.1006/jsre. 2001.6292

Pipinos, I. I, Judge, A. R., Selsby, J. T., Zhu, Z., Swanson, S. A., Nella, A. A., et al. (2007). The myopathy of peripheral arterial occlusive disease: part 1. Functional and histomorphological changes and evidence for mitochondrial dysfunction. Vasc. Endovascular Surg. 41, 481-489. doi: 10.1177/15385744073 11106

Pipinos, I. I, Judge, A. R., Selsby, J. T., Zhu, Z., Swanson, S. A., Nella, A. A., et al. (2008). The myopathy of peripheral arterial occlusive disease: part 2. Oxidative stress, neuropathy, and shift in muscle fiber type. Vasc. Endovascular Surg. 42, 101-112. doi: 10.1177/1538574408315995 
Pipinos, I. I, Judge, A. R., Zhu, Z., Selsby, J. T., Swanson, S. A., Johanning, J. M., et al. (2006). Mitochondrial defects and oxidative damage in patients with peripheral arterial disease. Free Radic. Biol. Med. 41, 262-269. doi: 10.1016/j.freeradbiomed.2006.04.003

Pipinos, I. I., Sharov, V. G., Shepard, A. D., Anagnostopoulos, P. V., Katsamouris, A., Todor, A., et al. (2003). Abnormal mitochondrial respiration in skeletal muscle in patients with peripheral arterial disease. J. Vasc. Surg. 38, 827-832. doi: 10.1016/S0741-5214(03)00602-5

Pipinos, I. I., Shepard, A. D., Anagnostopoulos, P. V., Katsamouris, A., and Boska, M. D. (2000). Phosphorus 31 nuclear magnetic resonance spectroscopy suggests a mitochondrial defect in claudicating skeletal muscle. J. Vasc. Surg. 31, 944-952. doi: 10.1067/mva.2000.106421

Rasmussen, U. F., Krustrup, P., Kjaer, M., and Rasmussen, H. N. (2003). Human skeletal muscle mitochondrial metabolism in youth and senescence: no signs of functional changes in ATP formation and mitochondrial oxidative capacity. Pflugers Arch. 446, 270-278. doi: 10.1007/s00424-003-1022-2

Regensteiner, J. G., Hargarten, M. E., Rutherford, R. B., and Hiatt, W. R. (1993a). Functional benefits of peripheral vascular bypass surgery for patients with intermittent claudication. Angiology 44, 1-10.

Regensteiner, J. G., Wolfel, E. E., Brass, E. P., Carry, M. R., Ringel, S. P., Hargarten, M. E., et al. (1993b). Chronic changes in skeletal muscle histology and function in peripheral arterial disease. Circulation 87, 413-421. doi: 10.1161/01.CIR.87.2.413

Robbins, J. M., Strauss, G., Aron, D., Long, J., Kuba, J., and Kaplan, Y. (2008). Mortality rates and diabetic foot ulcers: is it time to communicate mortality risk to patients with diabetic foot ulceration? J. Am. Podiatr. Med. Assoc. 98, 489-493. doi: 10.7547/0980489

Rolfe, D. F., and Brown, G. C. (1997). Cellular energy utilization and molecular origin of standard metabolic rate in mammals. Physiol. Rev. 77, 731-758.

Rooke, T. W., Hirsch, A. T., Misra, S., Sidawy, A. N., Beckman, J. A., Findeiss, L., et al. (2013). Management of patients with peripheral artery disease (compilation of 2005 and 2011 ACCF/AHA Guideline Recommendations): a report of the American College of Cardiology Foundation/American Heart Association Task Force on practice guidelines. J. Am. Coll. Cardiol. 61, 1555-1570. doi: 10.1016/j.jacc.2013.01.004

Ryan, T. E., Brophy, P., Lin, C. T., Hickner, R. C., and Neufer, P. D. (2014). Assessment of in vivo skeletal muscle mitochondrial respiratory capacity in humans by near-infrared spectroscopy: a comparison with in situ measurements. J. Physiol. 592, 3231-3241. doi: 10.1113/jphysiol.2014. 274456

Ryan, T. E., Schmidt, C. A., Alleman, R. J., Tsang, A. M., Green, T. D., Neufer, P. D., et al. (2016a). Mitochondrial therapy improves limb perfusion and myopathy following hindlimb ischemia. J. Mol. Cell. Cardiol. 97, 191-196. doi: 10.1016/j.yjmcc.2016.05.015

Ryan, T. E., Schmidt, C. A., Green, T. D., Spangenburg, E. E., Neufer, P. D., and McClung, J. M. (2016b). Targeted expression of catalase to mitochondria protects against ischemic myopathy in high-fat diet-fed mice. Diabetes 65:2553. doi: $10.2337 / \mathrm{db} 16-0387$

Ryan, T. E., Southern, W. M., Reynolds, M. A., and McCully, K. K. (2013). A cross-validation of near-infrared spectroscopy measurements of skeletal muscle oxidative capacity with phosphorus magnetic resonance spectroscopy. J. Appl. Physiol. 115, 1757-1766. doi: 10.1152/japplphysiol.00835.2013

Saks, V. A., Veksler, V. I., Kuznetsov, A. V., Kay, L., Sikk, P., Tiivel, T., et al. (1998). Permeabilized cell and skinned fiber techniques in studies of mitochondrial function in vivo. Mol. Cell. Biochem. 184, 81-100. doi: 10.1023/A:1006834912257

Schunk, K., Romaneehsen, B., Rieker, O., Duber, C., Kersjes, W., SchadmandFischer, S., et al. (1998). Dynamic phosphorus-31 magnetic resonance spectroscopy in arterial occlusive disease: effects of vascular therapy on spectroscopic results. Invest. Radiol. 33, 329-335. doi: 10.1097/0000 4424-199806000-00003

Sorlie, D., and Myhre, K. (1978). Effects of physical training in intermittent claudication. Scand. J. Clin. Lab. Invest. 38, 217-222. doi: 10.3109/00365 517809108414

Subherwal, S., Patel, M. R., Chiswell, K., Tidemann-Miller, B. A., Jones, W. S., Conte, M. S., et al. (2014). Clinical trials in peripheral vascular disease: pipeline and trial designs: an evaluation of the clinical trials.gov database. Circulation 130, 1812-1819. doi: 10.1161/CIRCULATIONAHA.114.011021
Szuba, A., Oka, R. K., Harada, R., and Cooke, J. P. (2006). Limb hemodynamics are not predictive of functional capacity in patients with PAD. Vasc. Med. 11, 155-163. doi: 10.1177/1358863x06074828

Thompson, J. R., Swanson, S. A., Haynatzki, G., Koutakis, P., Johanning, J. M., Reppert, P. R., et al. (2015). Protein concentration and mitochondrial content in the gastrocnemius predicts mortality rates in patients with peripheral arterial disease. Ann. Surg. 261, 605-610. doi: 10.1097/SLA.0000000000000643

Traupe, T., Ortmann, J., Stoller, M., Baumgartner, I., De Marchi, S. F., and Seiler, C. (2013). Direct quantitative assessment of the peripheral artery collateral circulation in patients undergoing angiography. Circulation 128, 737-744. doi: 10.1161/CIRCULATIONAHA.112.000516

Ueta, C. B., Gomes, K. S., Ribeiro, M. A., Mochly-Rosen, D., and Ferreira, J. C. B. (2017). Disruption of mitochondrial quality control in peripheral artery disease: new therapeutic opportunities. Pharmacol. Res. 115, 96-106. doi: 10.1016/j.phrs.2016.11.016

van Schaardenburgh, M., Wohlwend, M., Rognmo, Ø., and Mattsson, E. J. (2016). Mitochondrial respiration after one session of calf raise exercise in patients with peripheral vascular disease and healthy older adults. PLOS ONE 11:e0165038. doi: 10.1371/journal.pone.0165038

Wahl, D. G., Simon, J. P., Robin, B., Walker, P., Jouanny, P., Escanye, J. M., et al. (1994). Phosphorus magnetic resonance spectroscopy: a non-invasive technique for the study of occlusive arterial leg disease and peripheral vasodilator therapy. Angiology 45, 367-376. doi: 10.1177/000331979404500506

Wang, H., Hiatt, W. R., Barstow, T. J., and Brass, E. P. (1999). Relationships between muscle mitochondrial DNA content, mitochondrial enzyme activity and oxidative capacity in man: alterations with disease. Eur. J. Appl. Physiol. Occup. Physiol. 80, 22-27. doi: 10.1007/s004210050553

Wang, J. C., Criqui, M. H., Denenberg, J. O., McDermott, M. M., Golomb, B. A., and Fronek, A. (2005). Exertional leg pain in patients with and without peripheral arterial disease. Circulation 112, 3501-3508. doi: 10.1161/CIRCULATIONAHA.105.548099

Weiss, D. J., Casale, G. P., Koutakis, P., Nella, A. A., Swanson, S. A., Zhu, Z., et al. (2013). Oxidative damage and myofiber degeneration in the gastrocnemius of patients with peripheral arterial disease. J. Transl. Med. 11, 230-230. doi: 10.1186/1479-5876-11-230

West, A. M., Anderson, J. D., Epstein, F. H., Meyer, C. H., Hagspiel, K. D., Berr, S. S., et al. (2012). Percutaneous intervention in peripheral artery disease improves calf muscle phosphocreatine recovery kinetics: a pilot study. Vasc. Med. 17, 3-9. doi: $10.1177 / 1358863 \times 11431837$

White, S. H., McDermott, M. M., Sufit, R. L., Kosmac, K., Bugg, A. W., Gonzalez-Freire, M., et al. (2016). Walking performance is positively correlated to calf muscle fiber size in peripheral artery disease subjects, but fibers show aberrant mitophagy: an observational study. J. Transl. Med. 14:284. doi: 10.1186/s12967-016-1030-6

Yost, M. L. (2014). Cost-Benefit Analysis of Critical Limb Ischemia in the Era of the Affordable Care Act. Endovascular Today. Available online at: http://evtoday. com/2014/05/cost-benefit-analysis- of-critical-limb-ischemia-in-the-era- ofthe-affordable-care-act

Zatina, M. A., Berkowitz, H. D., Gross, G. M., Maris, J. M., and Chance, B. (1986). $31 \mathrm{P}$ nuclear magnetic resonance spectroscopy: non-invasive biochemical analysis of the ischemic extremity. J. Vasc. Surg. 3, 411-420. doi: 10.1016/07415214(86)90103-5

Ziegler-Graham, K., Mackenzie, E. J., Ephraim, P. L., Travison, T. G., and Brookmeyer, R. (2008). Estimating the prevalence of limb loss in the United States: 2005 to 2050. Arch. Phys. Med. Rehabil. 89, 422-429. doi: 10.1016/j.apmr. 2007.11.005

Conflict of Interest Statement: The authors declare that the research was conducted in the absence of any commercial or financial relationships that could be construed as a potential conflict of interest.

Copyright (C) 2017 Rontoyanni, Nunez Lopez, Fankhauser, Cheema, Rasmussen and Porter. This is an open-access article distributed under the terms of the Creative Commons Attribution License (CC BY). The use, distribution or reproduction in other forums is permitted, provided the original author(s) or licensor are credited and that the original publication in this journal is cited, in accordance with accepted academic practice. No use, distribution or reproduction is permitted which does not comply with these terms. 